Trauma Berufskrankh 2012 • 14[Suppl 2]:110-114 DOI 10.1007/s10039-011-1799-8

Online publiziert: 8. März 2012

(c) Springer-Verlag 2012
P. Gastmeier $\cdot$ A.-C. Breier $\cdot$ D. Sohr $\cdot$ C. Geffers

Institut für Hygiene und Umweltmedizin, Charité-Universitätsmedizin Berlin, Campus Benjamin Franklin

\title{
Prävention der postoperativen Wundinfektionen
}

\author{
Erkenntnisse aus 14 Jahren KISS \\ (Krankenhausinfektionssurveillancesystem)
}

Die Surveillance, d. h. die regelmäßige Analyse der auftretenden Wundinfektionen und die Ableitung von geeigneten Schlussfolgerungen, ist eine der wichtigsten Maßnahmen zur Prävention von Wundinfektionen. Dabei ist es besonders hilfreich, nicht nur die Entwicklung der Infektionsraten im eigenen Krankenhaus über die Zeit zu verfolgen, sondern sich auch mit anderen Krankenhäusern zu vergleichen, die dieselben Definitionen für postoperative Wundinfektionen verwenden und dieselben Bezugsgrößen berücksichtigen. Deshalb begann das Nationale Referenzzentrum (NRZ) für nosokomiale Infektionen im Jahr 1996, ein Krankenhausinfektionssurveillancesystem (KISS) für ausgewählte Infektionsarten in verschiedenen Risikopatientengruppen aufzubauen. Eines der ersten Module war das für die Surveillance von postoperativen Wundinfektionen bei Indikatoroperationen. Darunter sind auch einige Indikatoroperationen aus dem Bereich der Traumatologie/Orthopädie, wie Implantation von Hüft- und Knieendoprothesen, Reposition bei Fraktur des oberen Femurs und Eingriffe am oberen Sprunggelenk.

Die Zahl der auf freiwilliger Basis an KISS teilnehmenden Abteilungen nahm seit dessen Etablierung kontinuierlich zu. Im Zeitraum von 2006-2010 waren es insgesamt 616 Abteilungen aus 495 Krankenhäusern, darin enthalten sind 172 traumatologisch-orthopädische Abteilungen aus 142 Krankenhäusern.

\section{Methode}

Im Rahmen von KISS werden für die Diagnostik die Definitionen der CDC („Centers for Disease Control and Prevention") verwendet [6]. Sie unterscheiden nach oberflächlichen und tiefen Wundinfektionen sowie Infektionen von Organen und Körperhöhlen.

Alle neuen KISS-Teilnehmer werden ausführlich in die praktische Anwendung dieser Diagnosekriterien eingewiesen. Außerdem führt das NRZ jährliche Qualitätskontrollen zur Beurteilung der Diagnosegenauigkeit der Teilnehmer bei der Festlegung von postoperativen Wundin- fektionen durch. Diese erfolgen mit Hilfe von klinischen Fallbeispielen. Somit wird eine einheitliche und vergleichbare Diagnostik von postoperativen Wundinfektionen in KISS gewährleistet.

\section{Infektionsarten nach CDC}

\section{Postoperative oberflächliche Wundinfektion (A)}

Sie ist definiert als Infektion an der Inzisionsstelle innerhalb von 30 Tagen nach der Operation, die nur Haut oder subkutanes Gewebe mit einbezieht, und eines der folgenden Kriterien erfüllt:

\begin{tabular}{|llllll}
\hline \multicolumn{6}{l}{ Tab. 1 Rohe Wundinfektionsraten für Indikatorinfektionen } \\
\hline
\end{tabular}




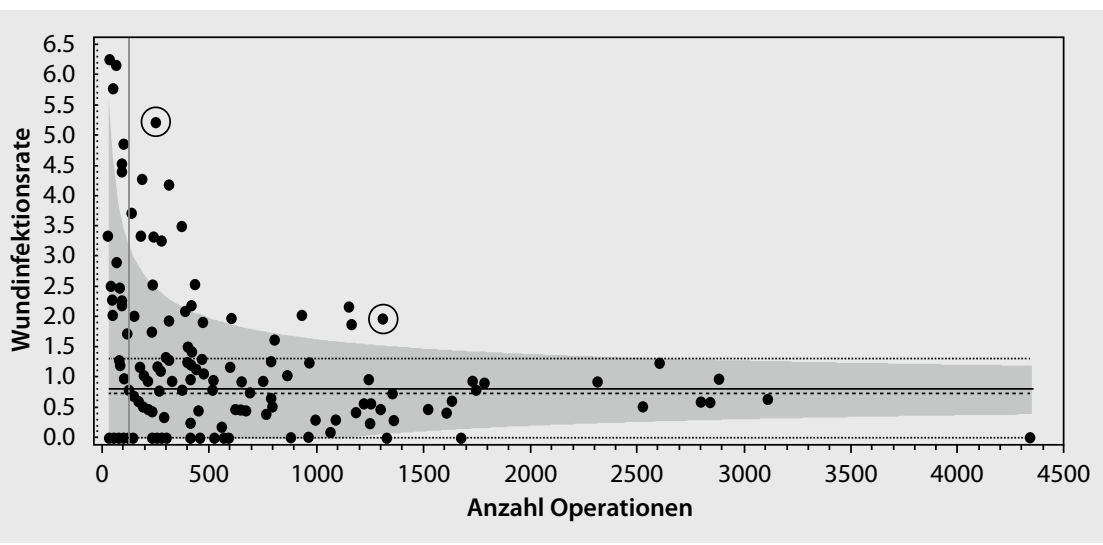

Abb. $1 \Delta$ Verteilung der Abteilungen bezüglich ihrer postoperativen Wundinfektionsraten nach Hüftendoprothesen bei Arthrose, 2006-2010, rechter unterer Kreis Abteilung A mit etwa 1300 Operationen; linker oberer Kreis Abteilung B mit nur etwa 200 Operationen, weitere Erläuterungen s. Text

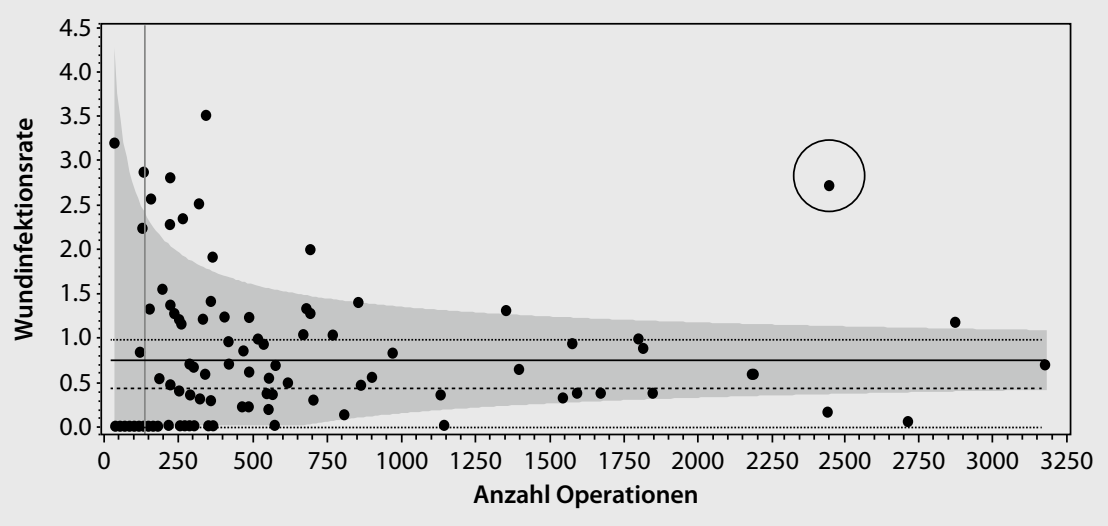

Abb. $2 \Delta$ Verteilung der Abteilungen bezüglich ihrer postoperativen Wundinfektionsraten nach Knieendoprothesen, 2006-2010, Kreis Abteilung mit signifikanter Ausreißerposition

1. eitrige Sekretion aus der oberflächlichen Inzision,

2. kultureller Nachweis von Erregern aus einem aseptisch entnommenen Wundsekret oder Gewebe von der oberflächlichen Inzision,

3. eines der folgenden Anzeichen:

- Schmerz oder Berührungsempfindlichkeit, lokalisierte Schwellung, Rötung oder Überwärmung, und

- bewusste Eröffnung der oberflächlichen Inzision durch den Chirurgen - dieses Kriterium gilt jedoch nicht bei Vorliegen einer negativen mikrobiologischen Kultur von der oberflächlichen Inzision,

4. Diagnose des behandelnden Arztes.

\section{Postoperative tiefe Wundinfektion (B)}

Sie wird diagnostiziert bei einer Infektion, die innerhalb von 30 Tagen nach der klinischen Untersuchung, während der erneuten Operation, bei der histopathologischen Befundung oder bei radiologischen Untersuchungen,

4. Diagnose des behandelnden Arztes.

\section{Infektion von Organen und Körper- höhlen im Operationsgebiet (C)}

Sie sind definiert als Infektionen, die innerhalb von 30 Tagen nach der Operation (innerhalb von $1 \mathrm{Jahr}$, wenn ein Implantat in situ belassen wurde) auftreten und mit der Operation in Verbindung zu stehen scheinen und Organe oder Körperhöhlen erfassen, die während der Operation geöffnet oder an denen während dieser manipuliert wurde, und die eines der folgenden Kriterien erfüllen:

1. eitrige Sekretion aus einer Drainage, die Zugang zu dem Organ bzw. der Körperhöhle im Operationsgebiet hat,

2. kultureller Nachweis von Erregern aus einem aseptisch entnommenen Wundsekret oder Gewebe aus einem Organ bzw. der Körperhöhle im Operationsgebiet,

3. Abszess oder sonstiges Zeichen einer Infektion des Organs bzw. der Körperhöhle im Operationsgebiet bei klinischer Untersuchung, während der erneuten Operation, bei der histopathologischen Befundung oder bei radiologischen Untersuchungen,

4. Diagnose des behandelnden Arztes. Implantat in situ belassen wurde) auftritt und mit dieser in Verbindung zu stehen scheint und die Faszienschicht und Muskelgewebe erfasst und eines der folgenden Kriterien erfüllt:

1. eitrige Sekretion aus der Tiefe der Inzision, aber nicht aus dem operierten Organ bzw. der Körperhöhle, da solche Infektionen zur Kategorie A3 gehören würden,

2. spontane oder vom Chirurgen bewusste Eröffnung, wenn der Patient mindestens eines der nachfolgenden Symptome zeigt: Fieber $\left(>38^{\circ} \mathrm{C}\right)$, lokalisierter Schmerz oder Berührungsempfindlichkeit - dieses Kriterium gilt jedoch nicht bei Vorliegen einer negativen mikrobiologischen Kultur aus der Tiefe der Inzision,

3. Vorhandensein eines Abszesses oder sonstiger Zeichen der Infektion, die tieferen Schichten betreffend, bei der

\section{Kasuistik}

Ein 79-jähriger Patient wurde nach Sturz mit Oberschenkelhalsbruch rechts aus einem Altenpflegeheim in ein Krankenhaus eingewiesen. Bei der Aufnahme wies das Altenheim auf eine MRSA-Besiedlung (MRSA: methicillinresistenter Staphylococcus aureus) des Patienten hin, worauf noch in der Notaufnahme Abstriche von Nase, Rachen, Perineum und Leisten entnommen wurden.

Die Entzündungsparameter lagen im Normbereich.

\section{Verlauf}

Tag 1 - Operation. Dem Patienten wurde rechts eine Hüfttotalendoprothese implantiert. Perioperativ wurde eine Antibiotikaprophylaxe durchgeführt. 
Tag 2. Der Patient war sehr verwirrt. Die Temperatur war unauffällig, die Wunde reizlos.

Tag 3. Das Ergebnis der Abstrichuntersuchung vom Aufnahmetag zeigte eine Besiedlung von Nase und Leiste mit MRSA.

In der Krankenakte findet sich der Eintrag: „Patient hat sich zum wiederholten Male den Verband entfernt und an den Drainagen manipuliert. Rezidivierende Verwirrtheitszustände.“

Tag 5. Die Wundränder waren stark gerötet und gespannt.

Tag 6. Durch den behandelnden Chirurgen wurde eine Abszessspaltung durchgeführt. Dabei erwies sich die Muskulatur als mit infiltriert. Zur mikrobiologischen Untersuchung wurde ein Wundabstrich entnommen.

Tag 8. Das Ergebnis der Wundabstrichuntersuchung von Tag 6 ergab eine Besiedlung mit MRSA. Es wurde eine lokale Wundbehandlung durchgeführt.

Tag 12. Es kam zu einer Besserung der Wundverhältnisse, und der Patienten wies ein zunehmend adäquates Verhalten auf.

Tag 19. Der Patient wurde bei gebessertem Allgemeinzustand in das Altenpflegeheim zurückverlegt. MRSA waren bis zur Entlassung immer wieder an verschiedenen Stellen nachweisbar.

\section{Diagnose}

Laut CDC-Definition handelte es sich um eine tiefe postoperative Wundinfektion.

\section{Auswertung - Surveillanceprotokoll}

Pro Indikatoroperationsart werden die sog. rohen Wundinfektionsraten berechnet (Anzahl Wundinfektionen pro 100 Operationen), zusätzlich werden die Daten stratifiziert nach oberflächlichen und tiefen Wundinfektionen bzw. Organinfektionen und nach der Anzahl der Risikopunkte, die sich aus dem Vorliegen eines hohen ASA-Scores ( $>2$, ASA: „American Society for Anesthesiologists“), einer langen Operationsdauer

Trauma Berufskrankh 2012 · 14[Suppl 2]:110-114

C) Springer-Verlag 2012

\section{P. Gastmeier · A.-C. Breier · D. Sohr · C. Geffers}

\section{Prävention der postoperativen Wundinfektionen. Erkenntnisse aus 14 Jahren KISS (Krankenhausinfektionssurveillancesystem)}

\section{Zusammenfassung}

Die Zahl der am Krankenhausinfektionssurveillancesystem (KISS) teilnehmenden Kliniken stieg seit dessen Aufbau in den letzten 14 Jahren stark an. Im Zeitraum 2006-2010 nahmen allein am Modul OP-KISS insgesamt 616 operative Abteilungen aus 495 Krankenhäusern auf freiwilliger Basis teil, darunter lieferten 172 Abteilungen Daten für Hüftendoprothesen bei Arthrose, 117 Abteilungen Daten für Hüftendoprothesen bei Fraktur und 107 Abteilungen Daten für Knieendoprothesen. Es konnte gezeigt werden, dass es möglich ist, durch gutes Feedback der Daten, geeignete Interpretation und Einleitung von Interventionen eine signifikante Reduktion der Infektionen zu erreichen. Signifikante Risikofaktoren für das Auftreten von
Wundinfektionen nach traumatologisch-orthopädischen Operationen sind männliches Geschlecht [OR=1,15 (OR:, Odds ratio")], Alter $(\mathrm{OR}=1,03)$, Wundkontaminationsklasse $(\mathrm{OR}=4,28)$, ASA-Score (ASA: „American Society of Anesthesiologists", OR=2,18) sowie die Jahreszeit Sommer $(\mathrm{OR}=1,30)$. Die häufigsten Erreger von Wundinfektionen bei traumatologisch-orthopädischen Operationen sind Staphylococcus aureus mit einem Anteil von $35,5 \%$, gefolgt von koagulasenegativen Staphylokokken $(12,9 \%)$ und Enterokokken $(12,8 \%)$.

\section{Schlüsselwörter}

Wundinfektion - Operationen - Surveillance . Epidemiologie $\cdot$ Risikofaktoren

\section{Prevention of surgical site infections. Results from 14 years of KISS (hospital infection surveillance system)}

\section{Abstract}

The number of hospitals participating in the German national nosocomial infection surveillance system (KISS) has been increasing during the last 14 years. A total of 616 surgical departments from 495 hospitals have provided data on a voluntary basis between 2006 and 2010, among them 172 departments with data on hip prosthesis following arthrosis, 117 departments with hip prostheses following fractures and 107 with knee prostheses. It was possible to demonstrate that feedback, careful interpretation of data and introduction of appropriate interventions lead to a significant reduction of surgical site infections. Significant risk factors for surgical site infections were male gender with an odds ratio (OR) of 1.15 , age $(\mathrm{OR}=1.03)$, wound contamination class $(\mathrm{OR}=4.28), \mathrm{ASA}$ (American Society of Anesthesiologists) score $(O R=2.18)$ and summer season $(\mathrm{OR}=1.30)$. The most frequent pathogens of surgical site infection were S.aureus (35.5\%) followed by coagulase negative staphylococci (12.9\%) and enterococci (12.8\%).

\section{Keywords}

Surgical site infection - Surgery - Surveillance . Epidemiology $\cdot$ Risk factors
(>75. Perzentile) und einer Wundkontaminationsklasse kontaminiert oder septisch ergeben. Zusätzlich wird für jede Abteilung bestimmt, wie hoch der Anteil der Wundinfektionen während des Krankenhausaufenthalts bzw. nach Entlassung war. Ferner wird die sog. standardisierte Wundinfektionszahl berechnet, die auch nach den wichtigsten Risikofaktoren adjustiert.

Das ausführliche Surveillanceprotokoll ist auf der Website http://www.nrz-hygiene.de $\mathrm{zu}$ finden.

\section{Aktuelle Surveillancedaten}

- Tab. 1 zeigt die Häufigkeit des Auftretens von postoperativen Wundinfektionen bei Indikatoroperationen aus dem Gebiet der Traumatologie/Orthopädie im Zeitraum von 2006-2010.

Dabei ergaben sich deutliche Unterschiede zwischen den teilnehmenden Abteilungen. Aus $\square$ Abb. 1 geht die Verteilung der Abteilungen bezüglich ihrer postoperativen Wundinfektionsraten nach Hüftendoprothesen bei Arthro- 
Tab. 2 Postoperative Wundinfektionsraten nach Hüft- und Knieendoprothesen. a (Nach [3])

\begin{tabular}{|c|c|c|c|c|c|c|c|c|}
\hline \multirow{2}{*}{$\begin{array}{l}\text { Teilnahme- } \\
\text { jahr }\end{array}$} & \multicolumn{4}{|c|}{ Hüftendoprothesen } & \multicolumn{4}{|c|}{ Knieendoprothesen } \\
\hline & $\begin{array}{l}\text { Abtei- } \\
\text { lungen }\end{array}$ & $\begin{array}{l}\text { Opera- } \\
\text { tionen }\end{array}$ & WI & WI-Rate (\%) & $\begin{array}{l}\text { Abtei- } \\
\text { lungen }\end{array}$ & $\begin{array}{l}\text { Opera- } \\
\text { tionen }\end{array}$ & WI & $\begin{array}{l}\text { WI-Rate } \\
\text { (\%) }\end{array}$ \\
\hline 1. Jahr & 14 & 4502 & 79 & 1,75 & 21 & 2664 & 27 & 1,01 \\
\hline 2. Jahr & 14 & 5365 & 81 & 1,51 & 21 & 2982 & 38 & 1,27 \\
\hline 3. Jahr & 14 & 5589 & 53 & 0,95 & 21 & 3365 & 25 & 0,74 \\
\hline Gesamt & & 15.456 & 213 & 1,38 & & 9011 & 90 & 1,00 \\
\hline
\end{tabular}

aWährend der ersten 3 Teilnahmejahre, nur Abteilungen mit kontinuierlicher Teilnahme über diese Zeit

Tab. 3 Häufigste Erreger von postope-

rativen Wundinfektionen in der Traumato-

logie/Orthopädie ${ }^{a}$

\begin{tabular}{ll} 
Erreger & $\begin{array}{l}\text { Häufigkeit pro 100 } \\
\text { postoperativen } \\
\text { Wundinfektionen }\end{array}$ \\
\hline Staphylococcus aureus & $\begin{array}{l}35,5 \text { (davon 19,1\% } \\
\text { MRSA) }\end{array}$ \\
\hline $\begin{array}{l}\text { Koagulasenegative } \\
\text { Staphylokokken }\end{array}$ & 12,9 \\
\hline $\begin{array}{l}\text { Enterococcus spp. } \\
12,8\end{array}$ \\
\hline $\begin{array}{l}\text { Escherichia coli } \\
\text { Enterobacter spp. }\end{array}$ & 3,6 \\
\hline $\begin{array}{l}\text { MRSA methicillinresistenter Staphylococcus } \\
\text { aureus. } \\
\text { aAuf der Basis von 2081 Wundinfektionen im Zeit- } \\
\text { raum 2006-2010 }\end{array}$ \\
\hline
\end{tabular}

Tab. 5 Anzahl der Abteilungen und Operationen und Verteilung der Operationsdauer. ${ }^{\text {a }}$ (Nach [4])

\begin{tabular}{|c|c|c|c|c|c|c|}
\hline \multicolumn{2}{|c|}{ Indikatoroperation } & \multirow[t]{2}{*}{$\begin{array}{l}\text { Teilnehmende } \\
\text { Krankenhäuser }\end{array}$} & \multirow[t]{2}{*}{$\begin{array}{l}\text { Opera- } \\
\text { tionen }\end{array}$} & \multicolumn{3}{|c|}{$\begin{array}{l}\text { Verteilung der Operationsdauer } \\
\text { (min) }\end{array}$} \\
\hline & & & & $\begin{array}{l}25 . \\
\text { Perzentile }\end{array}$ & Median & $\begin{array}{l}75 . \\
\text { Perzentile }\end{array}$ \\
\hline \multirow{2}{*}{$\begin{array}{l}\text { Hüftendo- } \\
\text { prothese }\end{array}$} & Arthrose & 80 & 57.993 & 61 & 80 & 101 \\
\hline & Fraktur & 66 & 12.926 & 60 & 75 & 95 \\
\hline \multicolumn{2}{|c|}{ Knieendoprothese } & 53 & 37.037 & 69 & 85 & 105 \\
\hline
\end{tabular}

se hervor, aus $\square$ Abb. 2 die entsprechende Verteilung bezüglich Knieendoprothesen. Durch die Darstellung als FunnelPlot wird berücksichtigt, dass die Wundinfektionsraten teilweise auf sehr großen Zahlen an operierten Patienten beruhen (z. B. Abteilung A mit etwa 1300 Operationen; unterer rechter Kreis in $\bullet$ Abb. 1), aber teilweise auch erst eine geringe Anzahl von Operationen für die Analyse berücksichtigt werden konnte (Abteilung $\mathrm{B}$ mit nur etwa 200 Operationen, oberer linker Kreis in Abb. 1). Die grau hinterlegte Fläche in Abb. 1 und 2 zeigt, dass die besonders hohen und besonders niedrigen Wundinfektionsraten durch Zufallsef-
Durch die langjährige Teilnahme vieler Abteilungen waren wir inzwischen immer wieder in der Lage, nachzuweisen, dass die Teilnahme in Zusammenhang mit einer gründlichen Analyse und der Einleitung geeigneter Intervention zu einer signifikanten Reduktion der Wundinfektionsraten führt. So konnten wir im Jahr 2005 auf der Basis der Daten von 14 Abteilungen, die kontinuierlich über mindestens 3 Jahre an KISS teilnahmen, eine signifikante Reduktion der Wundinfektionsrate um $46 \%$ [RR=0,54 (RR: relatives Risiko); 95\%-CI=0,38-0,77 (95\%CI: 95\%-Konfidenzintervall)] zeigen, die auch in der multivariaten Analyse unter Berücksichtigung der Risikofaktoren der Patienten bestätigt werden konnte. Auch bei Knieendoprothesen war diesbezüglich ein deutlicher Trend zu erkennen (• Tab. 2, [3]).

Auch für andere europäische Surveillancesysteme konnte gezeigt werden, dass durch regelmäßige Surveillance, Feedback und sinnvolle Interpretation der Infektionsraten signifikante Reduktionserfolge zu erreichen sind $[1,5]$.

\section{Epidemiologie von post- operativen Wundinfektionen}

Die meisten postoperativen Wundinfektionen in der Traumatologie/Orthopädie sind durch grampositive Erreger verursacht. Dabei hat Staphylococcus aureus mit 35,5\% zurzeit den höchsten Anteil, und etwa jeder fünfte Staphylococcus aureus, der eine Wundinfektion hervorruft, ist methicillinresistent (MRSA, $\bullet$ Tab. 3).

Nach den Ergebnissen einer vor kurzem durchgeführten multivariaten Risikofaktorenanalyse mittels GEE-Methode (GEE: „general estimating equation") und auf der Basis von KISS-Daten für den Zeitraum von 2005-2010, die 249.332 Operationen und 2441 Wundinfektionen aus 294 orthopädischen Abteilungen einschlossen, wurden für die Traumatologie/Orthopädie männliches Geschlecht, hohes Alter, ungünstige Wundkontaminationsklasse und ASA-Score sowie die Jahreszeit Sommer als signifikante Risikofaktoren für postoperative Wundinfektionen ermittelt (• Tab.4).

Selbstverständlich ist auch die Operationsdauer ein signifikanter Risikofak- 


\section{Hygiene: Heute und in Zukunft}

\begin{tabular}{|llll}
\hline $\begin{array}{l}\text { Tab. } 6 \\
\text { tionen. (Nach [7]) }\end{array}$ & Wundinfektionsrate in Abhängigkeit von der durchschnittlichen Anzahl der Opera- \\
\hline & $\begin{array}{l}\text { Postoperative Wundinfektionen pro } \\
\text { pro Jahr pro Abteilung }\end{array}$ & $>$ Operationen \\
\hline Anzahl der Operationen pro Jahr & $\leq 50$ & $\begin{array}{l}>50 \\
\leq 100\end{array}$ & $>100$ \\
\hline Hüftendoprothese (Arthrose) & 1,11 & 1,59 & 0,84 \\
\hline Knieendoprothese & 1,81 & 0,88 & 0,79 \\
\hline
\end{tabular}

tor, der in unserer Analyse aber nicht berücksichtigt wurde, weil eine vergleichsweise lange Operationsdauer zwar Surrogatparameter für komplizierte Operationsverhältnisse sein kann, aber auch durch die Technik des Operateurs und das Operationsmanagement beeinflusst wird. - Tab. 5 zeigt die Bandbreite der Operationsdauer für einige Operationsarten.

Auf gesundheitspolitische Ebene wird häufig diskutiert, ob Abteilungen mit einer hohen Fallzahl an Operationen einer bestimmten Art bessere Ergebnisse generieren. Dieser sog. „volume effect“ wurde auch auf der Basis der teilnehmenden Krankenhäuser für Hüft- und Knieendoprothesen untersucht, wobei 3 Kategorien ( $\leq 50$ Operationen, $>50-\leq 100$ Operationen und $>100$ Operationen) zugrunde gelegt wurden [7]. In diese Analyse gingen Daten von 206 Abteilungen, die in den Jahren 2003-2008 insgesamt 1.200.564 Operationen durchgeführt hatten, ein (• Tab. 6).

\section{Fazit für die Praxis}

\section{In Deutschland wurden nach Anga-} ben des statistischen Bundesamts im Jahr 2009 insgesamt 213.174 Hüft- sowie 159.137 Knieendoprothesen implantiert. Somit sind pro Jahr bundesweit etwa 3000 postoperative Wundinfektionen nach diesen Eingriffen zu erwarten. Legt man die in KISS nachgewiesenen Erfolge zur Reduktion von nosokomialen Infektionen zugrunde, kann man davon ausgehen, dass durch eine gute Surveillance inklusive Feedback und Intervention jährlich etwa 1000 Wundinfektionen nach Implantation von Hüft- und Knieendoprothesen vermieden werden können $[2,3]$.

\section{Korrespondenzadresse}

Prof. Dr. P. Gastmeier

Institut für Hygiene und Umweltmedizin, Charité-Universitätsmedizin Berlin, Campus Benjamin Franklin, Hindenburgdamm 27, 12203 Berlin

Petra.Gastmeier@charite.de

Interessenkonflikt. Der korrespondierende Autor gibt an, dass kein Interessenkonflikt besteht.

\section{Literatur}

1. Astagneau P, L'Heriteau F, Daniel F et al (2009) Reducing surgical site infection incidence through a network: results from the French ISO-RAISIN surveillance system. J Hosp Infect 72:127-134

2. Brandt C, Sohr D, Behnke M et al (2006) Reduction of surgical site infection rates with the help of benchmark data. Infect Control Hosp Epidemiol 27:1347-1351

3. Gastmeier P, Sohr D, Brandt C et al (2005) Reduction of orthopedic wound infections in 21 hospitals. Arch Orthop Trauma Surg 125:526-530

4. Gastmeier P, Sohr D, Breier AC et al (2011) Prolonged duration of operation: an indicator of complicated surgery or of surgical (mis)management? Infection 39(3):211-215

5. Geubbels E, Nagelkerke N, Mintjes-de Groot A et al (2006) Reduced risk of surgical site infections through surveillance in a network. Int J Qual Health Care 18:127-133

6. Horan T, Andrus M, Dudeck M (2008) CDC/NHSN surveillance definition of healthcare-associated infection and criteria for specific types of infections in the acute care setting. Am J Infect Control 36:309-332

7. Meyer E, Weitzel-Kage D, Sohr D, Gastmeier $P$ (2011) Impact of department volume on surgical site infections following arthrospoy, knee replacement or hip replacement. BMJ Qual Saf Jul 18. [Epub ahead of print] 